\title{
Non-contact vibration analysis using speckle-based techniques
}

\author{
I. Robles-Urquijo ${ }^{1}$, M. Lomer ${ }^{1,2 *}$, L. Rodriguez-Cobo ${ }^{1,2}$ and J.M. Lopez-Higuera ${ }^{1,2}$ \\ ${ }^{1}$ Photonics Engineering Group, University of Cantabria, 39005, Santander (Spain) \\ ${ }^{2}$ CIBER-bbn, Instituto de Salud Carlos III, 28029, Madrid (Spain)
}

\begin{abstract}
In this work, two different speckle based techniques are employed to quantify the movement of a vibrating surface. Directly projecting a coherent laser to a rough surface and collecting its associated subjective speckle using a high-speed camera, different frequencies are detected successfully. For the same purpose, a speckle pattern created by launching a coherent laser within a multimode fiber is employed as structured light to detect changes into the projection surface. The achieved results suggest that subjective speckle is better to analyze small sized areas, even at relative high frequencies, while projected speckle can give information about wider areas.
\end{abstract}

Keywords: Speckle interferometry, multimode fiber, vibration sensing

\section{INTRODUCTION}

Speckle patterns generated by the reflection of coherent light from a rough surface can be a very useful tool in metrology [1]. The different situations presented by a surface-moving target can be studied by changes in the speckle patterns recorded by a camera, where key parameters that represent the mechanical behavior of the target can be extracted without contact.

In industry, the vibration of a mechanical or electrical engine for example, can provide information on its operating status, useful and necessary data for maintenance, security or reliability [2]. Several optical methods have been proposed for measuring vibration including amplitude modulation or phase [3]. Among the presented techniques, interferometric systems exhibit high accuracy and resolution but may require many optical components [4]. On the contrary, the speckle interferometry, that is based on random interference of reflected light, is attracting interest on non-contact vibration measurements due to the useful benefits of combining optical techniques with interferometers: high sensitivity achieved with electromagnetic immunity. Particularly, two techniques can be employed to perform the addressed task based on speckle metrology: by capturing the direct speckle pattern created by illuminating the target with a laser, or analyzing the reflection on such target of a structured light projection from a multimode optical fiber. On both cases, for each element of the surface area resolved by the viewing system, these waves are added together vectorially, building a speckled intensity distribution over the surface, for which variations can be differentially processed [5] to perform frequency analysis of the desired target vibrations.

In this paper the vibration measurement without contact using two methods based on speckle patterns has been experimentally verified by two different methods: direct generation of the speckle pattern from the target surface and by analyzing the reflection of a speckle pattern (generated within a multimode fiber) projected to the same surface. The achieved results suggest a trade-off between technologies to analyze the desired surface.

\section{CAPTURE OF DIRECT SPECKLE AND FIBER PROJECTION}

When a coherent light source illuminates a surface, whose roughness exhibits a size comparable to that of the incoming light wavelength, a diffuse reflection process takes place, reflecting in many different ways the incoming light. This phenomenon, that is usually modelled as a random process, creates a very wide set of wave fronts that interfere between each other, obtaining a subjective speckle pattern that depends on the capturing device. However, for a given capturing scheme, any variation on the surface that creates the speckle pattern can be analyzed using the subjective pattern itself, achieving a long distance non-contact measurement system.

Another approach to analyze the vibration of a given surface can be achieved by using a structured light. Particularly, speckle patterns can be also employed as structured light, thus, launching a coherent laser source into a multimode fiber, projects a speckle pattern that can be employed to quantify the variations on a given surface. Depending on the properties of the laser source and those of the fiber employed to obtain the speckle pattern, a higher degree of granularity can be achieved, as well as being able to detect smaller perturbations within the surface to be analyzed.

*lomerm@ unican.es; phone +34 942200877; fax +34 942200877

25th International Conference on Optical Fiber Sensors, edited by Youngjoo Chung, Wei Jin,

Byoungho Lee, John Canning, Kentaro Nakamura, Libo Yuan, Proc. of SPIE Vol. 10323

103237W · @ 2017 SPIE · CCC code: 0277-786X/17/\$18 - doi: 10.1117/12.2265398 


\section{EXPERIMENTAL SETUP}

Both speckle-based approaches have been employed to analyze the vibration of a diffusive surface. A white surface target of 1 by $1 \mathrm{~cm}$ has been attached to a small speaker to provoke diffuse reflection, instead of the quasi-specular reflection of its metallic surface. The speaker has been excited with a sinusoidal wave form $(5 \mathrm{Vpp})$ of different frequencies using a signal generator. The two tested approaches to analyze this vibration are depicted in Fig 1.
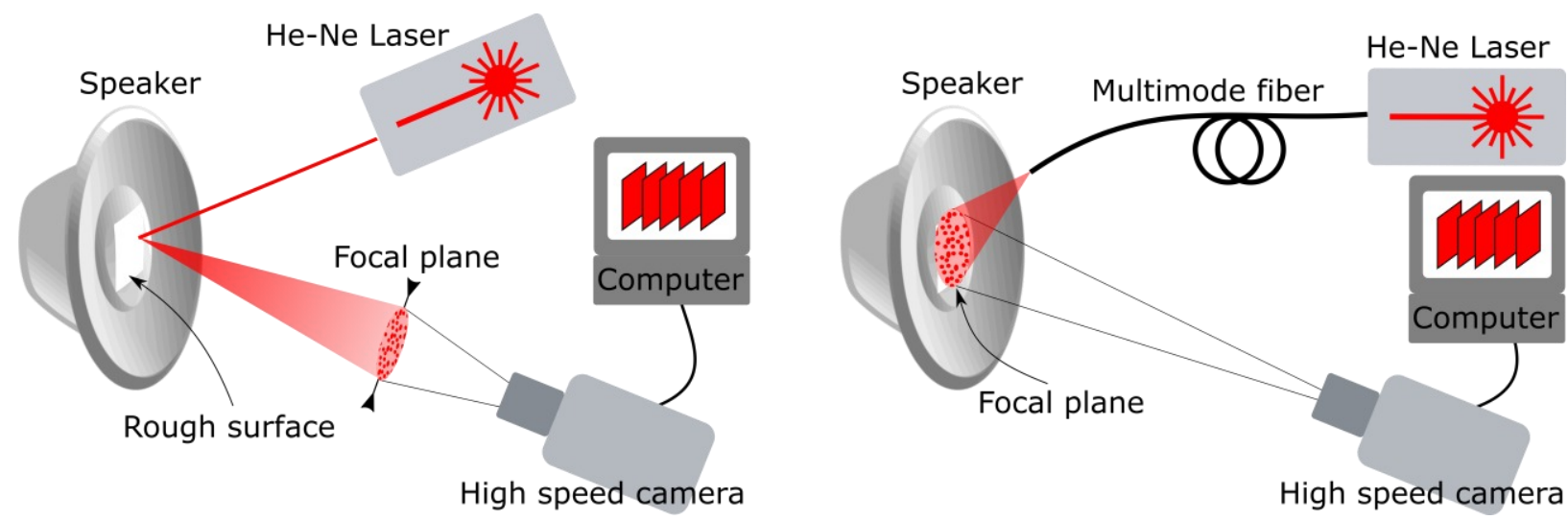

Figure 1. Scheme for testing both sensing approaches. A He-Ne laser directly illuminates a vibrating rough surface while its subjective speckle pattern is captured remotely (left). A He-Ne is guided through a multimode fiber ( 980 um core) to project a speckle pattern into the surface, which is then captured remotely by the camera (right).

Both test configurations exhibit different properties regarding their final performance. On one hand, projecting directly the coherent laser to a rough surface will produce a diffuse reflection creating a subjective speckle pattern that can be recorded by a high-speed camera (Fig. 1, left). As the focal plane of the camera optics is distanced (approx. $5 \mathrm{~cm}$ ) from the rough surface, the captured subjective speckle pattern becomes clearer, thus, it exhibits a very good sensitivity for the laser incident point (approx. 1mm diameter for the chosen He-Ne laser). On the other hand, a speckle pattern formed within a multimode fiber is launched to the rough surface to be directly collected by the same high-speed camera (Fig. 1, right). The projected pattern can be considered as structured light if no perturbation is applied to the multimode fiber, thus, any variation at the surface can be noticed in the reflected pattern. Therefore any vibration within the illuminated area (approx. $1 \mathrm{~cm} 2$ ) can be detected. An example of each speckle pattern is depicted in Fig. 2. The configuration of the camera is maintained constant for both tests.
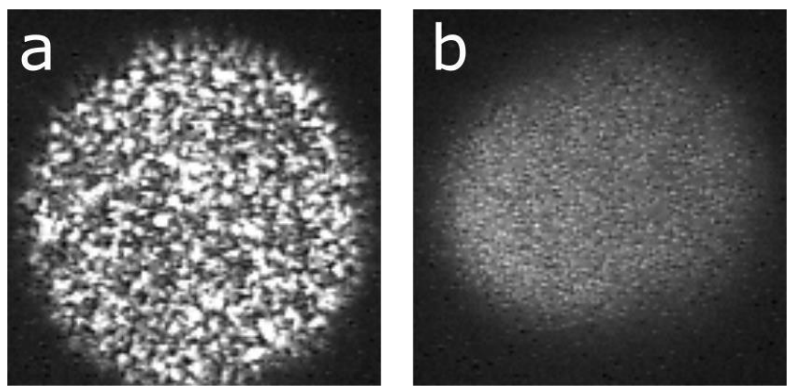

Figure 2. Example of 128 by 128 pixels speckle patterns captured for each setup. The subjective pattern is captured at $5 \mathrm{~cm}$ from the speaker surface (a). The reflection of the speckle pattern launched by the multimode fiber is captured placing the focal plane at the speaker surface (b).

For each configuration, a frequency sweep has been applied to the speaker while the signal amplitude was maintained (5 Vpp). The configuration of the camera has been maintained constant for all the experiments, achieving $150 \mathrm{fps}$, modifying only between the setups the focal point of the attached optics. As it can be observed in Fig. 2, the subjective speckle pattern obtained from the direct projection exhibits higher intensity values (a) than the reflection of the projected speckle pattern (b). 


\section{RESULTS}

The frame sequence for each experiment has been processed using a simple differential method. This method computes the intensity variation between speckles within the whole pattern between two consecutive frames [5]. This variation can be considered proportional to the perturbation when the variation between consecutive frames is small [5], that can be achieved for mechanical perturbations by increasing the acquisition rate. After stabilizing the frequency, a set of 900 frames has been captured for each setup and the most relevant results are depicted in Fig 3. Since the subjective speckle is captured directly from the diffuse reflection, the intensity of the pattern (Fig. 2, a) is better than on the projected speckle (Fig. 2, b). As described in [5], the speckle pattern intensity has influence on the final sensitivity using the differential processing scheme, as can be noticed in Fig. 3 (a, b), but it can be still employed for frequency detection.
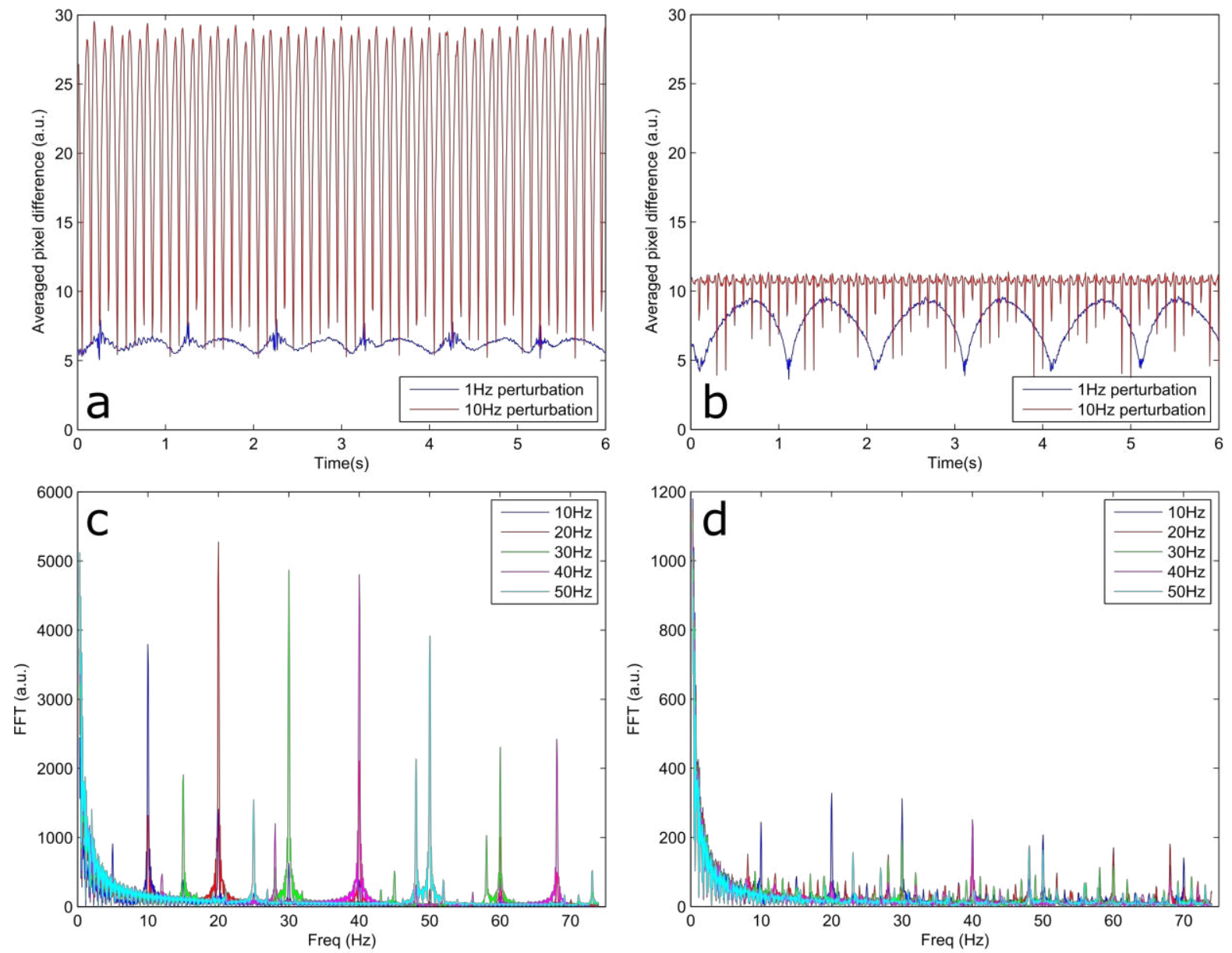

Figure 3. The most relevant results achieved during the frequency sweep. Differential sequences for the subjective speckle (a) and for the projected speckle (b) are depicted. FFT of the differential sequence for several frequencies computed for the subjective speckle (c) and projected speckle (d).

Based on the results depicted in Fig 3 (a), lower frequencies exhibit worse response for the subjective speckle method because the limitation for this scheme is the camera sampling frequency, since only the area illuminated with the laser is monitored: for low frequencies, the differential value between consecutive frames is very small. On the contrary, for higher frequencies (limited by the camera sampling rate), the differential value between frames is significant, improving the quality of the reconstructed sequence. The opposite circumstance can be appreciated in Fig.3 (b) when the differential sequence is computed from the projected speckle pattern. In this case, lower frequencies $(1 \mathrm{~Hz})$ exhibit better response than higher ones $(10 \mathrm{~Hz})$, despite the capturing rate being constant. This can be provoked by the projected speckle that illuminates a bigger area: the mechanical properties of a wider area of soft material have influence on the differential 
sequence (on the contrary than the small area illuminated in the other scenario, that can react "faster"). This situation can be verified when the excitation frequency is bigger.

In Fig. 3, the Fast Fourier Transform (FFT) of the sequences computed for different frequencies are depicted for the subjective speckle (c) and for the projected speckle (d). Apart from the harmonics, the FFT main peaks of the subjective speckle match perfectly to the excitation frequency up to $40 \mathrm{~Hz}$ (Fig. 3, c). However, on $50 \mathrm{~Hz}$ a secondary lobe provoked by the mechanical properties of the attached target and the surrounding noise (Fig. 3, c). This scenario is even worse when a large area of the target is analyzed by projecting the speckle pattern over all its area. In Fig. 3 (d), the only clearly detected frequency is $10 \mathrm{~Hz}$ (and its harmonics) while the others exhibit a much lower noise to signal ratio. Since both capturing conditions have been maintained, these results verify the difference on the mechanical properties along the target.

\section{CONCLUSIONS}

In this work, two different approaches based on speckle interferometry have been evaluated to analyze the movement of a vibrating surface. One approach employs the variations within the subjective speckle pattern created by diffuse reflection on a rough surface to quantify the movement. The other one employs a projected speckle pattern, generated within a multimode fiber as structured light, to analyze variations in the reflected speckle pattern. Depending on the frequency to be measured, the size of the speckle elements, the frame rate of the camera and the mechanical properties of the tested materials, both approaches can be valid, however the projected speckle covers a wider area, its final performance is more dependent on the mechanical properties of the tested surface and the sensitivity of the camera. On the contrary, when the subjective speckle is employed, a smaller area is under test (that covered by the laser spot), improving the spatial resolution and being less dependent on the mechanical properties of the vibrated area. Based on the achieved results, several configurations will be proposed to improve the performance of both systems.

\section{ACKNOWLEDGEMENT}

This work has been supported by the project TEC2013-47264-C2 and TEC2016-76021-C2 of the Spanish government.

\section{REFERENCES}

[1] R.S. Sirohi, Selected Papers on Speckle Metrology, SPIE Milestone Ser. MS 35, SPIE Optical Engineering Press, Washington, D.C., 1991.

[2] F. Chen, C.T. Griffen, T.E. Allen, "Digital speckle interferometry: some developments and applications for vibration measurement in the automotive industry", Opt. Eng. 37, 1390 (1998).

[3] F. Claveau, P. Fortier, S. Lord and D. Gingras, "Mechanical Vibration Analysis Using an Optical Sensor", Proceedings of CCECE 96, Calgary, 1996.

[4] W.C. Wang, C.H. Hwang, and S.Y. Lin, "Vibration measurement by the time-averaged electronic speckle pattern interferometry methods", Appl. Opt., 35(22), 4502 (1996).

[5] L. Rodriguez-Cobo, M. Lomer and J.M. Lopez-Higuera, "Fiber Specklegram Multiplexed Sensor", IEEE J. Lightwave Technol. IEEE, 33(12), 2591-2597 (2015). 